\title{
Phenotypic Characterization and Susceptibility Pattern of Gram Negative Bacterial Isolates from Cases of Central Line Associated Blood Stream Infections in a Tertiary Care Institute
}

\author{
Akhilesh, P.S. Tomar ${ }^{*}$, Anjali Kushwah ${ }^{2}$ and Harshada Shah ${ }^{3}$ \\ ${ }^{1}$ Department of Microbiology, NSCB Medical College, Jabalpur (MP)-482003, India \\ ${ }^{2}$ Department of Pharmacology, G R Medical College, Gwalior (MP), India \\ ${ }^{3}$ Department of Microbiology, R D Gardi Medical College, Ujjain (MP), India \\ *Corresponding author
}

Keywords

Central Line

Associated Blood

Stream Infections,

Gram negative

bacterial infection,

Multi Drug

Resistant.

\section{Article Info}

Accepted:

04 July 2017

Available Online:

10 September 2017
A B S T R A C T

Resistant and specially Multi Drug Resistant (MDR) Gram negative isolates from the cases of Central Line Associated Blood Stream Infections (CLABSI) are a serious challenge for the treatment to clinicians. Present study was undertaken for phenotypic characterization and susceptibility pattern of Gram negative bacterial isolates from cases of CLABSI in a tertiary care institute. Materials this descriptive cross sectional hospital based study was conducted in a tertiary care teaching hospital over a period of one and half year from January 2012 to June 2013. Centers for Disease Control and Prevention (CDC ${ }^{\mathrm{TM}_{\mathrm{S}} \text { ) }}$ CLABSI case definitions were used to label a case as CLABSI. Only culture proven cases, out of clinically suspected was included in the study for evaluation. Incidence Central Line Associated Blood Stream Infections were $(0.31 \%)$ with 38 culture positive cases. The overall rate of CLABSI was 14.64 per 1000 device days with (0.04) device utilization ratio. Gram negative isolates predominated (79\%) over Gram positive isolates (21). Among Gram negative bacterial isolates from CLABSI, Klebsiella pneumoniae (40\%) was the commonest followed by E. coli $(26.66 \%)$. \%). Rising trends of antimicrobial resistance for common Gram negative bacterial isolates were noticed. Continuous surveillance of CLABSI and microbiological reporting of culture isolates is a need of time in the health care facility.

\section{Introduction}

Hospitalized patients suffered by the most common complications during their hospital stay are Health care-associated infections (HAIs). Despite of significant advances in infection control policies, HAIs remain a major public health problem and a significant cause of raised morbidity and mortality among hospitalized patients. ${ }^{1}$ Among several factors responsible for institutional morbidity and mortality Central line associated bloodstream infection (CLABSI) is a major contributing factor and consequently it is also responsible for longer hospital stay and increased expenditure on treatment. ${ }^{1}$ Insertion of Central Venous Catheter in a chronically ill ICU admitted patients is a common practice now a days worldwide. CVC inserted in emergency without following proper aseptic procedure, for longer duration and daily manipulation of $\mathrm{CVC}$ for the purpose of 
administration of fluids, drugs, and blood products are responsible for higher rate of CLABSI in ICU settings. ${ }^{1}$ Higher rates of infection often leads to increased rates of antimicrobial prescription which in turn contribute to increased antimicrobial resistance. ${ }^{2}$ Treatment of multi-drug resistant pathogens is a costly affair. ${ }^{2}$ Data regarding the incidence, prevalence, epidemiology, aetiology and susceptibility pattern of isolates are frequently available from the resource rich countries but same is not true for resource poor countries. ${ }^{3-8}$ Both gram positive and gram negative organisms are responsible for CLABSI. Gram positive cocci (both Staphylococcus aureus and the coagulasenegative staphylococci) are the leading causes of CLABSI followed by Gram negative bacilli. ${ }^{9}$ Multidrug-resistant organisms are increasingly being reported. ${ }^{10}$

The present study was designed to study the phenotypic characterization and susceptibility of gram negative bacteria from Central Line Associated Blood Stream Infections (CLABSI) in our geographic area. The purpose of the Antimicrobial Susceptibility Test (ABST) was;

To guide the choice of the antimicrobial for the treatment.

To provide surveillance data to monitor the resistance trend.

\section{Material and Methods}

This descriptive cross sectional hospital based study was conducted in a tertiary care teaching hospital over a period of one and half year from January 2012 to June 2013. The study was carried out in surgical ICU, general surgery, and obstetrics/ gynecology wards. Patients admitted in hospital and fitting in the definition of HAI were included in the study. CDC's CLABSI case definitions ${ }^{11}$ were used to label a case as CLABSI. Only culture proven cases, out of clinically suspected was included in the study for evaluation. Samples (CVC tip and Blood) were collected from suspected CLABSI patients. Both active and passive surveillance methods were used for sample collection. Active surveillance was done by visiting various study areas daily along with infection control nurse. Passive surveillance was done by following the positive culture results obtained in microbiology laboratory to retrospective wards in the hospital. The labeled specimens were transported to microbiology laboratory within $30 \mathrm{~min}$ of collection. Specimen were inoculated on appropriate culture media including blood agar, MacConkey agar, chocolate agar and incubated for $16-18 \mathrm{hrs}$ at $35-37^{\circ} \mathrm{C}$ by using standard laboratory techniques. ${ }^{12,13}$

Identification of bacteria was based on the colony characteristics of the organism i.e., colony morphology, hemolysis on blood agar, changes in the physical appearance of the differential media and enzyme activities of the organisms, Gram staining and biochemical tests. 12,13 Antimicrobial sensitivity was performed on Muller Hinton agar plates by Kirby-Bauer disk diffusion method as per CLSI guidelines. ${ }^{12-14}$ Antibiotic discs were procured from HiMedia laboratories, Mumbai, India.

Isolates were labeled susceptible, resistant and intermediate on the basis of CLSI disc zone interpretative criterion. ${ }^{14}$ All Gram negative isolates were tested for ESBL and MBL production as per CLSI guidelines. ${ }^{12-14}$ ESBL positive Klebsiella pneumonia ATCC 700603 and ESBL negative Escherichia coli ATCC 25922 were included in the study for quality control of ESBL tests. ${ }^{14}$ Pseudomonas aeruginosa 27853 was used as the control for MBL tests. ${ }^{14}$ All the media and reagents were procured from Himedia, Mumbai, India. 


\section{Results and Discussions}

Presenting data is a part of a prospective cross sectional study in which we assessed prevalence of various HAIs and phenotypic characterization of Gram negative bacterial isolates along with their susceptibility profile. A total of 18536 patients were admitted in the wards under surveillance (surgery wards, obstetrics/ gynecology wards and medical ICU) for HAI during one and half year period (Jan 2012 - June 2013). 6526 patients were excluded from the study because they had a length of hospital stay of less than 48 hours.

The remaining 12010 admissions formed study population. The incidence of health care associated infections was $(5.14 \%)$ and Central Line Associated Blood Stream Infections contributed $(0.31 \%)$ with 38 culture positive cases. The overall rate of CLABSI was 14.64 per 1000 device days with (0.04) device utilization ratio (Table 1). Among Gram negative bacterial isolates from CLABSI, Klebsiella pneumoniae $(40 \%)$ was the commonest followed by E. coli $(26.66 \%)$ and Pseudomonas aeruginosa (20\%) (Table 2).
Rest was Gram positive bacterial isolates. Gram negative isolates predominated $(79 \%=$ 30 out of 38) over Gram positive isolates $(21 \%=8$ out of 38$)$ (Table 2). Among common Gram negative bacterial isolates Klebsiella pneumoniae exhibited (100\%) resistance to ampicillin, amoxicillin + clavulanic and cefuroxime followed by $(91.66 \%)$ to cefotaxime, cefazolin and ceftazidime, $(83.33 \%)$ to piperacillin and piperacillin + Tazobactum, $(75 \%)$ to cefepime, gentamicin and ciprofloxacin $(58.33 \%)$ to amikacin and none of the isolates showed resistance against imipenem. E. coli exhibited $(100 \%)$ resistance to ampicillin, followed by $(87.5 \%)$ to amoxicillin + clavulanic, piperacillin and cefuroxime, $(75 \%)$ to cefotaxime, ceftazidime and ciprofloxacin, (50\%) to gentamicin and none of the isolates showed resistance against imipenem and amikacin. Pseudomonas aeruginosa exhibited (50\%) resistance to ciprofloxacin, followed by $(33.33 \%)$ to piperacillin and piperacillin and piperacillin + Tazobactum, (16.66\%) to Amikacin, and none of the isolates showed resistance against imipenem and gentamicin (Table 3).

Table.1 Device utilization ratio and incidence and of CLABSI

\begin{tabular}{lcccccc}
\hline $\begin{array}{c}\text { Type of } \\
\text { HAI }\end{array}$ & $\begin{array}{c}\text { Type of } \\
\text { device }\end{array}$ & $\begin{array}{c}\text { Device- } \\
\text { days } \\
\text { number }\end{array}$ & $\begin{array}{c}\text { Patients } \\
\text { days } \\
\text { number }\end{array}$ & $\begin{array}{c}\text { Device } \\
\text { utilization } \\
\text { ratio }\end{array}$ & $\begin{array}{c}\text { Culture positive } \\
\text { DAI } \\
\text { number }\end{array}$ & $\begin{array}{c}\text { Rate per 1000 } \\
\text { device-days }\end{array}$ \\
\hline CLABSI* & $\mathrm{CVC}^{*}$ & 2594 & 61491 & 0.04 & 38 & 14.64 \\
\hline *Central & & & & & & \\
\hline
\end{tabular}

Table.2 Gram negative bacterial isolates from CLABSI $(n=30)$

\begin{tabular}{lcc}
\hline Microorganisms & Number & $(\boldsymbol{\%})$ \\
\hline Klebsiella pneumoniae & 12 & 40 \\
E. coli & 08 & 26.66 \\
Pseudomonas aeruginosa & 06 & 20 \\
Citrobacter freundii & 01 & 3.33 \\
Citrobacter koseri & 01 & 3.33 \\
Enterobacter aerogenes & 01 & 3.33 \\
Klebsiella oxytoca & 01 & 3.33 \\
\hline
\end{tabular}


Table.3 Resistance pattern of common Gram negative bacterial isolates from CLABSI (\% resistance)

\begin{tabular}{lccc}
\hline Antibiotics & $\begin{array}{c}\text { Klebsiella pneumoniae } \\
(\mathbf{n = 1 2})\end{array}$ & E. coli $(\mathbf{n = 0 8})$ & $\begin{array}{c}\text { Pseudomonas } \\
\text { aeruginosa }(\mathbf{n}=\mathbf{0 6})\end{array}$ \\
\hline Ampicillin & $12(100)$ & $08(100)$ & $\mathrm{ND}$ \\
Amoxycillin + Clavulanic acid & $12(100)$ & $07(87.5)$ & $\mathrm{ND}$ \\
Piperacillin & $10(83.33)$ & $07(87.5)$ & $02(33.33)$ \\
Piperacillin + Tazobactum & $10(83.33)$ & $04(50)$ & $02(33.33)$ \\
Cefazolin & $11(91.66)$ & $07(87.5)$ & $\mathrm{ND}$ \\
Cefepime & $09(75)$ & $06(75)$ & $01(16.66)$ \\
Cefotaxime & $11(91.66)$ & $06(75)$ & $\mathrm{ND}$ \\
Cefoxitin & $08(66.66)$ & $05(62.5)$ & $01(16.66)$ \\
Ceftazidime & $11(91.66)$ & $06(75)$ & $00(00)$ \\
Cefoparazone & $\mathrm{ND}$ & $\mathrm{ND}$ & $01(16.66)$ \\
Cefuroxime & $12(100)$ & $07(87.5)$ & $\mathrm{ND}$ \\
Imipenem & $00(00)$ & $00(00)$ & $00(00)$ \\
Amikacin & $07(58.33)$ & $00(00)$ & $01(16.66)$ \\
Gentamicin & $09(75)$ & $04(50)$ & $00(00)$ \\
Ciprofloxacin & $09(75)$ & $06(75)$ & $03(50)$ \\
Trimethoprim/Sulfamethoxazole & $08(66.66)$ & $01(12.5)$ & $\mathrm{ND}$ \\
\hline
\end{tabular}

\section{Observations}

Nosocomial infections acquired due to insertion of devices are serious cause of concern to the health care facility. ${ }^{15,16}$ Data obtained from surveillance of device associated infections are of great help to the clinicians in treatment of such infections and for health care settings to develop effective infection control policies. To the best of my knowledge there has been very little information available on health care setting DAI rate in the literature from India. ${ }^{16}$ In order to determine CLABSI, a total of 2594 central venous catheter days, in 287 cases of central venous catheterization in various wards and ICUs were studied in present study. CLABSI was confirmed microbiologically in 38 cases out of a total 98 clinically suspected cases. The study showed a lower use of CVC (0.04 vs. 0.50) compared with the device utilization reported by the US in the NNISS network ${ }^{15}$ and (0.70) by INICC. ${ }^{6}$ The percentage distribution of CLABSI among HAI was $(6.15 \%)$ which was lower than INICC overall CLABSI $(30 \%)^{6}$ and $(61.3 \%)$ by A. Mehta et al., ${ }^{6}$ The overall rate of CLABSI was $0.31 \%$ or 14.64 per 1000 device days. This is quite high in comparison to other Indian, US and Turkish studies, $6,15,17$ while the rate is comparable with the overall INICC rate of 12.5 per 1000 device days. ${ }^{6}$ Antimicrobial resistance is a worldwide problem that needs urgent attention. Though this is a worldwide scenario, but situation in India is vulnerable because of injudicious use and over the counter availability of antimicrobials. $^{18,19}$ Insertion of among chronically ill patients have become necessary tool for the successful treatment. In the present study, K. pneumoniae $(40 \%)$ was the most common isolate followed by $E$. coli (26.66\%) and Pseudomonas aeruginosa (20\%). Results are not consistent with the findings of other observers. ${ }^{8}, 20-23$ All the isolates of $K$. pueumoniae were resistant to ampicillin and amoxicillin/clavulanic acid. Amikacin was resistant to $(58.33 \%)$ isolates and was lowest among all commonly used antibiotics. Similar resistance reported by Datta et al., $(59 \%){ }^{24}$ Fortunately no resistance was observed for carbapenems in isolates of $K$. pneumoniae. This signifies the prudent use of this group in future in study 
setup. Hundered percent isolates of E. coli were resistant to ampicillin. While no resistance was found to carbapenems and amikacin in this study. These results are not in match with Datta et al., who showed (10\% and 57\%) resistance to carbapenems and amikacin respectively. ${ }^{24}$ Among Pseudomonas aeruginosa no resistance was found for ceftazidime, carbapenems and gentamicin. Different hospital setup, use of antibiotics and infection control policies among different institutes may be the reasons for varied results.

Data from present study indicates that resistance for most of the classes of antibiotics has increased up to the alarming level. Timely action and continuous surveillance of CLABSI and susceptibility pattern of isolates is a need of time for the better management of CLABSI cases and for the development of better infection control policies.

\section{References}

1. Rello J, Ochagavia A, Sabanes E, Roque M, Mariscal D, Reynaga E, et al. Evaluation of outcome of intravenous catheter-related infections in critically ill patients. Am J Respir Crit Care Med 2000; 162 (3 Pt 1):1027-30.

2. Pittet, D. et al. Infection control as a major World Health Organization priority for developing countries. J Hosp Infect. 2008;68:285-92.

3. Edwards JR, Peterson KD, Andrus ML, Tolson JS, Goulding JS, Dudeck MA, et al. National Healthcare Safety Network (NHSN) Report, data summary for 2006, issued June 2007. Am J Infect Control 2007;35:290-301.

4. Vincent JL, Bihari DJ, Suter PM, Bruining HA, White J, Nicolas-Chanoin $\mathrm{MH}$, et al; EPIC International Advisory Committee. The prevalence of nosocomial infection in intensive care units in Europe: results of the European Prevalence of Infection in Intensive Care (EPIC) Study. JAMA 1995;274:639-44.

5. Safdar N, Crnich CJ, Maki DG. Nosocomial infections in the intensive care unit associated with invasive medical devices. Curr Infect Dis Rep 2001;3:48795.

6. Mehta A, Rosenthal VD, Mehta Y, Chakravarthy M, Todi SK, Sen N, et al. Device-associated nosocomial infection rates in intensive care units of seven Indian cities. Findings of the International Nosocomial Infection Control Consortium (INICC). J Hosp Infect 2007;67:168-74.

7. Kaur R, Mathai AS, Abraham J. Mechanical and infectious complications of central venous catheterisations in a tertiary-level intensive care unit in northern India. Indian $J$ Anaesthesia 2012;56:376-81.

8. Patil HV, Patil VC, Ramteerthkar MN, Kulkarni RD. Central venous catheterrelated bloodstream infections in the intensive care unit. Indian $J$ Crit Care Med 2011;15:213-23.

9. Beekmann SE, Henderson DK. Infections caused by percutaneous intravascular devices. In: Mandell GL, Bennett JE, Dolin R, eds. Principles and Practice of Infectious Disease. Vol 1. $6^{\text {th }}$ ed. Philadelphia, PA: Elsevier; 2005:33473361.

10. de Lissovoy, G. et al. Surgical site infection: incidence and impact on hospital utilization and treatment costs. Am J Infect Control. 2009;37:387-97.

11. Garner JS, Jarvis WR, Emori TG, Horan TC, Hughes JM. CDC definitions for nosocomial infections, 1988. Am J Infect Control 1988;16:128-40.

12. Collee GJ, Marimon BP, Frazer AG, Simmons A Makie McCartney Practical Medical Microbiology. Collee JG, 14ed; 11:245-258, 4:151-177. 
13. Koneman EW, Allen S, Janda W, Schreckenberger P, Winn WC. Color Atlas and Text book of Diagnostic Microbiology, $6^{\text {th }}$ edn. New York : Lippincott; 2006.

14. CLSI. Performance Standards for Antimicrobial Susceptibility Testing; Twentieth Informational Supplement. CLSI document M100-S20. Wayne, PA: Clinical and Laboratory Standards Institute; 2010.

15. National Nosocomial Infections Surveillance System. National Nosocomial Infections Surveillance (NNIS) System Report, data summary from January 1992 through June 2004, issued October 2004. Am. J. Infect. Control. 2004;32:470-85.

16. Singh S, Pandya Y, Patel R, Paliwal M, Wilson A, Trivedi S. Surveillance of device-associated infections at a teaching hospital in rural Gujrat- India. Indian Journal of Medical Microbiology. 2010;28:342-7.

17. Inan D, Saba R, Yalcin An, Yilmaz M, Ongut G, Ramazanoglu A, et al. Deviceassociated nosocomial infection rates in Turkish medical-surgical intensive care units. Infect Control Hosp Epidemiol. 2006;27:343-8.

18. Wattal C. "Antibiotic policy": why and for whom. JIMSA. 2004;17:170-73.

19. Wattal C, Goel N, Oberoi Jk, Raveendran R, Datta S, Prasad KJ. Surveillance of
Multidrug Resistant Organisms in a Tertiary Care Hospital in Delhi, India. JAPI. 2010;58:32-36.

20. Rosenthal VD, Maki DG, Salomao R, et al. Device-associated nosocomial infections in 55 intensive care units of 8 developing countries. Ann Intern Med. 2006;145:582-91.

21. Moonens F, el Alami S, Van Gossum A, Struelens MJ, Serruys E. Usefulness of gram staining of blood collected from total parenteral nutrition catheter for rapid diagnosis of catheter related sepsis. J Clin Microbiol. 1994;32:1578-9.

22. Velasco E, Thuler LC, Martins CA, Nucci M, Dias LM, Gonçalves VM, et al. Epidemiology of bloodstream infections at a cancer center. Sao Paulo Med J. 2000;118:131-8.

23. Schwaber MJ, Krasner CN, Gold HS, Venkataraman L, Avigan DE, Karchmer AW, et al. Detection of Staphylococcus aureus in peripheral blood stem cell cultures after sterilization of standard blood cultures. J Clin Apher. 2003;1:379.

24. Datta S, Wattal C, Goel N, Oberoi JK, Raveendran R, Prasad KJ. A ten year analysis of multi-drug resistant blood stream infections caused by Escherichia coli \& Klebsiella pneumoniae in a tertiary care hospital. Indian J Med Res. 2012;135: 907-12.

\section{How to cite this article:}

Akhilesh, P.S. Tomar, Anjali Kushwah and Harshada Shah. 2017. Phenotypic Characterization and Susceptibility Pattern of Gram Negative Bacterial Isolates from Cases of Central Line Associated Blood Stream Infections in a Tertiary Care Institute. Int.J.Curr.Microbiol.App.Sci. 6(9): 75-80. doi: https://doi.org/10.20546/ijcmas.2017.609.008 